\title{
The simplest shadowing
}

\author{
by JERzY OMBACH (Kraków)
}

\begin{abstract}
Two different and easy proofs are presented that a hyperbolic linear homeomorphism of a Banach space admits the shadowing.

In this note we establish two different proofs of the Shadowing Theorem in the simplest but nontrivial situation. Namely, we show that any linear homeomorphism of a Banach space which is hyperbolic has also the shadowing property. The most common version of the Shadowing Theorem is a basic result in the theory of smooth dynamical systems and it says that a dynamical system defined by a diffeomorphism or a vector field on a compact manifold has the shadowing property on its hyperbolic set. There are a number of proofs of this theorem, all rather difficult and long (see for example [4], [8], [5], [1]); however, an idea behind each of them is quite readable. In the linear case the ideas are similar yet the proofs are much simpler. Therefore this note may be of some interest for beginners in dynamical systems and nonspecialists. The first proof we establish is inspired by a work of Katok [4] and it also works for noninvertible maps. The second proof has perhaps a more geometric character. The first half of it is due to Morimoto and this half is sufficient for the whole proof in the finite-dimensional case. Also, the second half suffices in that situation. In the infinite-dimensional case, however, they should be combined together. For simplicity we consider only discrete time linear systems; for continuous time systems (i.e. given by systems of linear differential equations) our proofs also work. Our result is the following
\end{abstract}

THEOREM. A hyperbolic linear homeomorphism of a Banach space has the shadowing property.

Let $(X, d)$ be a metric space. Let $f: X \rightarrow X$ be a map. We define the

1991 Mathematics Subject Classification: Primary 58F99.

Key words and phrases: hyperbolic, shadowing, pseudo-orbit, contraction, expansive. Supported by Grant IM PB 658/2/21. 
$n$th iteration of $f$ as $f^{0}=$ identity, $f^{n+1}=f^{n} \circ f$. We define an orbit to be a sequence $\left\{x_{n}\right\}_{n \in \mathbb{Z}}$ satisfying

$$
x_{n+1}=f x_{n}
$$

for all $n \in \mathbb{Z}$. If $f$ is a homeomorphism then an orbit is a sequence $\left\{f^{n} x\right\}_{n \in \mathbb{Z}}$. If a sequence $\left\{x_{n}\right\}_{n \in \mathbb{Z}}$ satisfies the above equality up to some perturbation we say it is a pseudo-orbit. More precisely, we say that a sequence $\left\{x_{n}\right\}_{n \in \mathbb{Z}}$ is a $\delta$-pseudo-orbit if

$$
d\left(f x_{n}, x_{n+1}\right) \leq \delta
$$

for all $n \in \mathbb{Z}$. Now, we say that a map $f$ has the pseudo-orbits tracing property, or the shadowing property if for every $\varepsilon>0$ there exists $\delta>0$ such that any $\delta$-pseudo-orbit $\left\{x_{n}\right\}_{n \in \mathbb{Z}}$ is $\varepsilon$-traced by some genuine orbit $\left\{y_{n}\right\}_{n \in \mathbb{Z}}$, i.e.

$$
d\left(x_{n}, y_{n}\right) \leq \varepsilon
$$

for all $n \in \mathbb{Z}$.

Let $X$ be a Banach space, and $f: X \rightarrow X$ a linear continuous map. We say that $f$ is hyperbolic if the spectrum of $f, \sigma(f)$, is disjoint from the unit circle $S^{1}$. It is a classical result (see [2] for example) that the hyperbolicity is equivalent to the following condition. There exists a decomposition $X=$ $X_{1} \oplus X_{2}$ and $f=f_{1} \oplus f_{2}$, where $f_{i}: X_{i} \rightarrow X_{i}$ are linear continuous, $i=1,2$, and there exist norms $\|\cdot\|_{i}$ on $X_{i}, i=1,2$, such that: (i) $\left\|f_{1}\right\|_{1}<1$, $f_{2}$ is a homeomorphism and $\left\|f_{2}^{-1}\right\|_{2}<1$. (ii) The norm $\|\cdot\|$ defined by $\|x\|=\left\|x_{1}\right\|_{1}+\left\|x_{2}\right\|_{2}$ is equivalent to the original norm of $X$. If $f$ is a homeomorphism then so is $f_{1}$.

The following two lemmas will be used in the proofs of the Theorem. Their proofs are straightforward.

LEMMA 1. Let $\left(X_{i}, d_{i}\right), i=1,2$, be metric spaces and $f_{i}$ maps on $X_{i}$. Let $X=X_{1} \times X_{2}$ be equipped with a metric generating the product topology. Let $f=f_{1} \times f_{2}$ be a map on $X$ defined by $f\left(x_{1}, x_{2}\right)=\left(f_{1}\left(x_{1}\right), f_{2}\left(x_{2}\right)\right)$. Then $f$ has the shadowing property if and only if $f_{1}$ and $f_{2}$ do.

LEMMA 2. Let $f$ be a homeomorphism of a metric space $X$ such that $f$ and its inverse $f^{-1}$ are uniformly continuous. Then $f$ has the shadowing property if and only if $f^{-1}$ does.

Remark 1. Note that in order to prove the Theorem, it suffices to prove that if a linear map $f$ is a contraction, i.e. $\|f\|<1$, then $f$ has the shadowing property. In fact, if we have proved this and then assume that $f$ is hyperbolic, then $f=f_{1} \oplus f_{2}$ where both $f_{1}$ and $f_{2}^{-1}$ are contractions and so have the shadowing property. By Lemma $2, f_{2}$ has this property, and by Lemma 1 , so does $f$. 
Proof of the Theorem. We prove more:

TheOREM A. A hyperbolic continuous linear map of a Banach space has the shadowing property.

By Remark 1, Theorem A is a simple consequence of the following general result:

Proposition 1. Let $X$ be a complete metric space and $f: X \rightarrow X a$ contraction, i.e. there exists a constant $a<1$ such that

$$
d(f x, f y) \leq a d(x, y)
$$

for all $x, y \in X$. Then $f$ has the shadowing property.

Proof. Fix $\varepsilon>0$ and define $\delta=(1-a) \varepsilon$. Let $\mathbf{x}=\left\{x_{n}\right\}_{n \in \mathbb{Z}}$ be a $\delta$-pseudo-orbit. Define a metric space $\mathbf{E}$ by

$$
\mathbf{E}=\left\{\mathbf{y}: \mathbf{y}=\left\{y_{n}\right\}_{n \in \mathbb{Z}}, d\left(x_{n}, y_{n}\right) \leq \varepsilon\right\}
$$

with metric

$$
D(\mathbf{y}, \mathbf{z})=\sup \left\{d\left(y_{n}, z_{n}\right): n \in \mathbb{Z}\right\} .
$$

It is easy to see that $(\mathbf{E}, D)$ is complete. For $\mathbf{y} \in \mathbf{E}$ define a sequence $\mathbf{F}(\mathbf{y})$ by

$$
\mathbf{F}(\mathbf{y})_{n}=f y_{n-1} \quad \text { for } n \in \mathbb{Z} .
$$

For all $n \in \mathbb{Z}$ we have

$$
\begin{aligned}
d\left(f y_{n-1}, x_{n}\right) & \leq d\left(f y_{n-1}, f x_{n-1}\right)+d\left(f x_{n-1}, x_{n}\right) \\
& \leq a d\left(y_{n-1}, x_{n-1}\right)+\delta \leq a \varepsilon+(1-a) \varepsilon=\varepsilon,
\end{aligned}
$$

which means that $\mathbf{F}(\mathbf{E}) \subset \mathbf{E}$. Also, we can easily see that $\mathbf{F}$ is a contraction with contraction constant $a$, since

$$
\begin{aligned}
D(\mathbf{F}(\mathbf{y}), \mathbf{F}(\mathbf{z})) & =\sup \left\{d\left(f y_{n-1}, f z_{n-1}\right): n \in \mathbb{Z}\right\} \\
& \leq a \sup \left\{d\left(y_{n-1}, z_{n-1}\right): n \in \mathbb{Z}\right\} \\
& =a \sup \left\{d\left(y_{n}, z_{n}\right): n \in \mathbb{Z}\right\} \leq a D(\mathbf{y}, \mathbf{z}) .
\end{aligned}
$$

The Banach Contraction Principle completes the proof of Proposition 1, and thus of the Theorem.

Second proof of the Theorem. We will work with semi-orbits and semi-pseudo-orbits. A sequence $\left\{x_{n}\right\}_{n \geq 0}$ is a positive orbit if (1) holds for all $n \geq 0$, and it is a positive $\delta$-pseudo-orbit if (2) holds for such $n$ 's. A sequence $\left\{x_{n}\right\}_{n \leq 0}$ is a negative orbit (negative $\delta$-pseudo-orbit) if the corresponding conditions hold for all $n \leq 0$. Now, one can define the shadowing ${ }^{+}$ (shadowing $^{-}$) property in the obvious way.

Proposition 2. Let $X$ be a metric space (not necessarily complete) and $f: X \rightarrow X$ a contraction with contraction constant $a<1$. Let $\beta>0$. Then 
for any positive $\beta$-pseudo-orbit $\left\{x_{n}\right\}_{n \geq 0}$ and every $x \in X$ with $d\left(x_{0}, x\right) \leq \beta$ we have

$$
d\left(f^{n} x, x_{n}\right) \leq \frac{\beta}{1-a} \quad \text { for all } n \geq 0 .
$$

This means that $f$ has the shadowing ${ }^{+}$property; moreover, a positive pseudo-orbit is shadowed by any positive orbit starting close to the first point of the pseudo-orbit.

Proof. For any $n \geq 1$ we have

$$
d\left(f^{n} x, x_{n}\right) \leq d\left(f^{n} x, f x_{n-1}\right)+d\left(f x_{n-1}, x_{n}\right) \leq a d\left(f^{n-1} x, x_{n-1}\right)+\beta .
$$

So, by induction we get

$$
d\left(f^{n} x, x_{n}\right) \leq a^{n} \beta+a^{n-1} \beta+\ldots+\beta,
$$

which proves (4).

Morimoto [6] proved the Theorem for $X=\mathbb{R}^{n}$. The main step of his proof is the following.

Proposition 3. Let $X$ be a Banach space and $f: X \rightarrow X$ an expansive (i.e. $f^{-1}$ is contractive) linear homeomorphism. Then $f$ has the shadowing ${ }^{+}$ property.

Proof. Put $a=\left\|f^{-1}\right\|<1$. Fix $\delta>0$ and a positive $\delta$-pseudo-orbit $\left\{x_{n}\right\}_{n \geq 0}$. So we have

$$
x_{n+1}=f x_{n}+h_{n} \quad \text { with }\left\|h_{n}\right\| \leq \delta \text { for all } n \geq 0 .
$$

Simple induction shows that for $n \geq 1$,

$$
x_{n}=f^{n} x_{0}+f^{n-1} h_{0}+\ldots+h_{n-1}
$$

and hence

$$
x_{n}=f^{n}\left(x_{0}+\sum_{i=1}^{n} f^{-i} h_{i-1}\right) .
$$

Define

$$
y_{0}=x_{0}+\sum_{i=0}^{\infty} f^{-i} h_{i-1} .
$$

The series is convergent as $\left\|f^{-i} h_{i-1}\right\| \leq a^{i} \delta$. We have

$$
\begin{aligned}
\left\|x_{n}-f^{n} y_{0}\right\| & =\left\|f^{n}\left(x_{0}+\sum_{i=0}^{n} f^{-i} h_{i-1}\right)-f^{n}\left(x_{0}+\sum_{i=0}^{\infty} f^{-i} h_{i-1}\right)\right\| \\
& \leq \sum_{i=n+1}^{\infty}\left\|f^{n-i} h_{i-1}\right\| \leq \delta \sum_{i=n+1}^{\infty} a^{i-n}=\frac{\delta a}{1-a} \leq \varepsilon
\end{aligned}
$$

for any $\varepsilon>0$ if $\delta$ is small enough. 
Second proof of the Theorem (end). According to Remark 1 we have to prove that a contractive linear homeomorphism $f$ has the shadowing property.

Set $a=\|f\|$ and fix $\varepsilon>0$. Let $\varepsilon_{1}=(1-a) \varepsilon$, let $\delta_{1}$ correspond to $\varepsilon_{1}$ by the shadowing ${ }^{+}$property of $f_{1}$ and put $\delta=\delta_{1} a^{-1}$. Let $\left\{x_{n}\right\}_{n \in \mathbb{Z}}$ be a $\delta$-pseudo-orbit.

We show, by shadowing ${ }^{+}$of $f^{-1}$ (Proposition 3 ), that the negative part of this pseudo-orbit is $\varepsilon_{1}$-shadowed by some point and then, by Proposition 2 , this point also shadows the positive part of the pseudo-orbit. More precisely, we put $z_{n}=x_{-n}$ for $n \geq 0$ and have

$$
\left\|f^{-1} z_{n}-z_{n+1}\right\| \leq\|f\|\left\|x_{-n}-f x_{-n-1}\right\| \leq a \delta \leq \delta_{1} .
$$

By the shadowing ${ }^{+}$property of $f^{-1}$ we get a sequence $\left\{w_{n}\right\}_{n \geq 0}$ such that

$$
\left\|w_{n}-z_{n}\right\| \leq \varepsilon_{1} \text { and } w_{n}=f w_{n+1} \text { for all } n \geq 0 .
$$

In particular, $\left\|w_{0}-x_{0}\right\| \leq \varepsilon_{1}$, hence by (4) and the choice of $\varepsilon_{1}$ we have $\left\|f^{n} w_{0}-x_{n}\right\| \leq \varepsilon$ for all $n \geq 0$. Putting

$$
y_{n}= \begin{cases}w_{-n} & \text { for } n \leq 0, \\ f^{n} w_{0} & \text { for } n \geq 0,\end{cases}
$$

we get an orbit which $\varepsilon$-traces $\left\{x_{n}\right\}_{n \in \mathbb{Z}}$.

Remark 2. If the space $X$ is of finite dimension then, as shown in [6] (see also [7]), shadowing ${ }^{+}$implies shadowing. Hence, taking into account Remark 1 we see that just one of the two propositions 2 or 3 , yields the proof of the Theorem.

Remark 3. If the space is finite-dimensional then the converse to the Theorem is also true, namely the shadowing property of a linear isomorphism of $\mathbb{R}^{n}$ implies its hyperbolicity (see [3], [7]).

\section{References}

[1] R. Bowen, Equilibrium States and the Ergodic Theory of Anosov Diffeomorphisms, Lecture Notes in Math. 470, Springer, 1975.

[2] M. C. Irwin, Smooth Dynamical Systems, Academic Press, 1980.

[3] S. Kakubari, A note on a linear homeomorphism on $\mathbb{R}^{n}$ with the pseudo-orbit tracing property, Sci. Rep. Niigata Univ. Ser. A 23 (1987), 35-37.

[4] A. Katok, Local properties of hyperbolic sets (in Russian), appendix to the Russian edition of: Z. Nitecki, Differentiable Dynamics, Mir, Moscow, 1975.

[5] K. Meyer and G. Sell, An analytic proof of the Shadowing Lemma, Funkc. Ekvac. 30 (1987), 127-133.

[6] A. Morimoto, Some stabilities of group automorphisms, in: Manifolds and Lie Groups, Progr. Math. 14, Birkhäuser, 1981, 283-299. 
[7] J. Ombach, The Shadowing Lemma in the linear case, Univ. Iagell. Acta Math., to appear.

[8] M. Shub, Global Stability of Dynamical Systems, Springer, 1987.

INSTITUTE OF MATHEMATICS

JAGIELLONIAN UNIVERSITY

REYMONTA 4

30-059 KRAKÓW, POLAND

Reçu par la Rédaction le 16.3.1992 